\title{
Presence of illegal drugs in drivers involved in fatal road traffic accidents in Spain
}

\author{
M. Carmen del Río, F. Javier Alvarez* \\ Drugs and Alcohol Research Group, Department of Pharmacology and Therapeutics, Faculty of Medicine, University of Valladolid, \\ 47005 Valladolid, Spain
}

Received 24 November 1998; received in revised form 12 March 1999; accepted 30 April 1999

\begin{abstract}
This study investigated the presence of illegal drugs in the blood of 285 fatally injured drivers in Spain. Illegal drugs were detected in $10.2 \%$ of all samples. Illicit drugs alone were detected in $2.5 \%$ and together with other substances in $7.7 \%$. Cocaine was the most common drug detected. The mean number $( \pm$ S.D.) of substances detected was $2.6 \pm 1.2$ : consisting of 46 illegal drugs, 14 alcohol cases and 16 medicines. Three concentration levels of the different substances have been established: low, medium and high-toxic. In $68.9 \%$ of the samples in which an illegal drug was detected, a substance was also found at the high-toxic level. The results show that illegal drugs are commonly detected in road accident victims. (C) 2000 Elsevier Science Ireland Ltd. All rights reserved.
\end{abstract}

Keywords: Illegal drugs; Alcohol; Road traffic accidents; Spain

\section{Introduction}

In recent years the role played by illicit drugs in road safety has become the object of increasing interest. Among illegal drugs are included groups which are quite diverse in respect of their effects on the central nervous system, psychomotor performance, and risktaking behaviour, together with other aspects. Generally speaking, and when these aspects are analysed jointly, it is considered that the effects of these drugs impair the ability to drive safely (Del Río and Alvarez, 1995a). Whilst the relationship between alcohol, impaired driving ability and accident risk is well established, in the case of different illegal drugs there are many points which require clarification. The hypothesis that drug-taking drivers are at greater risk in terms of accident involvement has not yet been satisfactorily determined (Albery et al., 1998; Consensus Development Panel, 1985; Road Safety Committee, 1996).

Within the European Union, the different member states have legislation concerning two aspects: firstly,

\footnotetext{
* Corresponding author. Tel.: + 34-983-423077; fax: + 34-983423022 .

E-mail address: alvarez@med.uva.es (F. Javier Alvarez)
}

driving under the influence of different illegal drugs is forbidden, and, secondly, in accordance with Council Directive 91/439/EEC, stipulation is made that a driving licence will not be issued to anyone who abuses, is dependent upon, or has a disorder brought about by illegal drugs (Albery and Strang, 1995; Del Río and Alvarez, 1995a). It is very difficult, however, to put this legislation into practice.

Recent reports emphasise various aspects concerning illicit drugs and driving about which we have little information. In this regard we should mention the recent review from Albery et al. (1998) on the matter, as well as the studies conducted for the Pompidou Group (Council of Europe) by Krüger et al. (1998) on the legal provisions relating to illicit drugs and road traffic accidents, and those by De Gier (1998) on the prevalence of illicit drugs in road traffic accidents. Also, there is now available the literature review on illicit drugs and driving carried out by the European Monitoring Centre for Drugs and Drug Addiction (1999).

If determining the effect of an illegal drug on driving ability is complex in the laboratory, it is even more so in a real-life driving situation. In addition, apart from the effect of a drug on psychomotor performance and ability to drive, there are other factors related to drug- 
taking patterns which influence the final effect the drug will have on driving ability: these include dosage, how the drug is administered, the taking of different substances simultaneously (even with alcohol), associated morbidity, overdose and withdrawal symptoms, etc. (Del Río and Alvarez, 1995a).

The actual importance of driving whilst under the effects of illicit drugs is not fully defined. It has been estimated that around $10 \%$ of all people killed or injured in road traffic accidents were taking some sort of psychotropic medication that may have been a contributory factor (De Gier, 1993).

In studies from various countries concerning fatally injured drivers, the incidence of illegal drugs is variable as is the frequency of the different substances detected (7-30\%); the most commonly detected illegal drug is cannabis (Budd et al., 1989; Gjerde et al., 1993; Ferrara et al., 1994; Drummer, 1995; Logan and Schwilke, 1996; Merced and Jeffery, 1995; Hansen et al., 1996; Christophersen and Morland, 1997; Harmeet et al., 1997; Charlier and Plomteux, 1998).

In Spain, a previous study carried out on 1500 drivers showed that 3\% had driven under the effects of illegal drugs during the previous 12 months (Del Río and Alvarez, 1995b). According to a study carried out by the National Institute of Toxicology on 250 drivers involved in fatal road accidents, illegal drugs, and in particular cocaine, were detected in $8 \%$ of them (Rams et al., 1993).

This study was undertaken to assess the presence of alcohol, illegal and medicinal drugs in people involved in fatal road accidents in Spain. The article is specifically concerned with illegal drugs and has as its aims: (i) to analyse the presence of illegal drugs in fatally injured drivers, (ii) to assess their presence alone or in combination with other substances, and (iii) to quantify the substances detected.

\section{Methods}

It was stated in this study that all information obtained would be anonymous and used for scientific and not legal purposes. The target population consisted of drivers killed in road accidents. The data available in each case was: sex, age, day and time of the accident. Samples of venous blood were taken within $8 \mathrm{~h}$ of the victim's death and were later sent to our centre.

Samples concerning 285 road accident victims from January 1994 to October 1996 in the north of Spain were obtained. Two hundred and fifty-five $(89.5 \%)$ out of the 285 samples analysed were men and $30(10.5 \%)$ were women. Ninety-six subjects $(33.7 \%)$ were people belonging to the $16-25$ age group, $129(45.3 \%)$ were between 26 and 45 years of age and $60(21.0 \%)$ were aged over 45. One hundred and thirteen samples
$(39.6 \%)$ represented drivers fatally injured on working days (Monday to Friday) and $172(60.4 \%)$ on nonworking days (Saturday and Sunday). The average age $( \pm$ S.D. $)$ was $34.1 \pm 13.2(33.9 \pm 13.1$ for men and $36.0 \pm 14.7$ for women). Of the samples obtained on non-working days $46 \%$ represented persons under the age of 26 , whilst on working days the figure was $29 \%$.

All samples were also screened for the presence of drugs other than alcohol (medicines and illegal drugs) by immunological or chromatographic methods as appropriate. Positive results after screening were confirmed by alternative methods (GC/MS, HPLC or GC methods) and the concentrations of the psychoactive drugs or metabolites were determined. Blood samples were analyzed for alcohol (ethanol) by head space gas chromatography (Peat and Finkle, 1992; Tedeschi et al., 1992).

The substances other than alcohol were classified into two groups: illegal drugs, and medicines. Within the first group, categorization into the following groups was established: amphetamine, opiates, cocaine, cannabis, synthetic drugs (ecstasy) and hallucinogenic drugs. Within the second group, different sub-groups were established, in accordance with the Anatomical Therapeutic Chemical Classification system. For the present study, benzodiazepines were always considered medicinal drugs. Once a substance had been detected, the samples were classified for the purpose of this study into the following groups: (i) illegal drugs, (ii) illegal drugs with alcohol, (iii) illegal drugs with medicines, (iv) illegal drugs plus alcohol plus medicines, and (v) no alcohol-no drugs.

Alcohol, illegal drugs and medicines were categorized and, the rating used was based on three levels: low, medium and high concentration (low therapeutic, therapeutic, and high non-therapeutic concentration for medicines), such as has previously been used in other studies (Drummer, 1994). For example, for cocaine, these categories have been established as: level low $(<0.1 \mathrm{mg} / \mathrm{l})$, level-II $(0.1-0.25 \mathrm{mg} / \mathrm{l})$ and level-III ( $>$ $0.25 \mathrm{mg} / \mathrm{l}$ ). In relation to alcohol, these categories have been arbitrarily established as: level-I or low (0.01-0.49 $\mathrm{g} / 1)$, level-II or medium $(0.5-0.99 \mathrm{~g} / \mathrm{l})$ and level-III or high-toxic $(\geq 1 \mathrm{~g} / \mathrm{l})$.

Statistical analysis of the data was conducted in the Data Processing Centre at Valladolid University. Statistical analysis was by means of SAS software version 6.07 (SAS Institute SAS). $P$-values $<0.05$ were considered to be significant differences.

\section{Results}

Table 1 shows the presence of the different substances found in Spanish drivers killed in road traffic accidents. In $40 \%(n=114)$ of the cases no substance 
Table 1

Prevalence of drugs in fatally injured drivers in Spain

\begin{tabular}{lrr}
\hline & $n$ & $\%$ \\
\hline No substance & 114 & 40.0 \\
Some substance & 171 & 60.0 \\
$\quad$ Alcohol & 144 & 50.5 \\
Illegal drugs & 29 & 10.2 \\
$\quad$ Alone & 7 & 2.5 \\
$\quad$ +Alcohol & 12 & 4.2 \\
$\quad$ Medicines & 8 & 2.8 \\
$\quad$ Medicines + alcohol & 2 & 0.7 \\
Medicines & 26 & 9.1 \\
\hline
\end{tabular}

was detected, whilst in $60 \%$ some substance was found. Alcohol was found in $50.5 \%(n=144)$, some medication in $9.1 \%(n=26)$ and some illicit drugs were found in $10.2 \%(n=29)$.

Regarding illicit drugs (Table 1), these substances alone were detected in 2.5\% $(n=7)$, and illegal drugs with other substances in 7.7\% $(n=22)$ : of these, illegal drugs with alcohol were detected in $4.2 \%(n=12)$, with medicines in $2.8 \%(n=8)$, and with medicines and alcohol in $0.7 \%(n=2)$.

Cocaine, $7.4 \%(n=21)$, was the most frequently detected drug, followed by opiates, $4.9 \%(n=14)$, amphetamine and cannabis, $1.4 \%(n=4)$, and synthetic drugs, $1.1 \%(n=3)$. Table 2 shows their distribution in accordance with some sociodemographic characteristics; no differences were observed $(P>0.5)$ with regard to sex, age groups or days of the week.

In the 29 samples in which some illegal drug was detected, a total of 76 substances were encountered (mean $2.6 \pm 1.2$ ): of these 46 were illegal drugs, 14 alcohol and 16 medicines (11 benzodiazepines and five analgesics-anti-inflammatory).
Of these 46 illegal drugs, in $91.3 \%$ of cases the drug was accompanied by another substance, and in only four cases $(8.7 \%)$ was it detected alone (Table 3). Of these drugs $19.6 \%$ were found in combination with alcohol only, $43.5 \%$ with substances other than alcohol, and $28.3 \%$ with alcohol plus another substance. It should be mentioned that the most commonly detected illegal drug in combination with alcohol was cocaine, in $57.1 \%$ of cases. In addition, opiates were generally found in combination with substances other than alcohol, in $78.6 \%$ of cases. Of the 13 cases encountered of opiates in combination with other substances, nine were associated with an analgesic or a benzodiazepine drug.

As stated above, categorization of the different substances was based on three degrees or levels. Of the 46 substances classified as illegal drugs, $63 \%(n=29)$ were ranked in group or level-I, $19.6 \%$ in level-II $(n=9)$ and $27.6 \%(n=8)$ in level-III. Opiates were predominant in level-III, $45.7 \%(n=5)$. Of all samples analyzed (29), illegal drugs were found in $72.4 \%(n=21)$ in group or level-I, in $41.4 \%(n=9)$ in level-II, and in $27.6 \%(n=8)$ in level-III (Table 4).

In addition, of the 29 samples in which some type of illegal drug was detected, in 20 of these some kind of substance was found with level-III (illegal drug level-III eight cases, alcohol level-III nine cases, and medicinal drug level-III three cases) (Table 4); in the remaining nine cases, six were found with some type of level-II substance, and in the other three all were level-I substances.

\section{Discussion}

This study reveals how often illegal drugs are found in people killed in road accidents, with a figure of $10.2 \%$ being detected in the samples analysed. Likewise,

Table 2

Sociodemographic characteristics of testing positive for illegal drugs

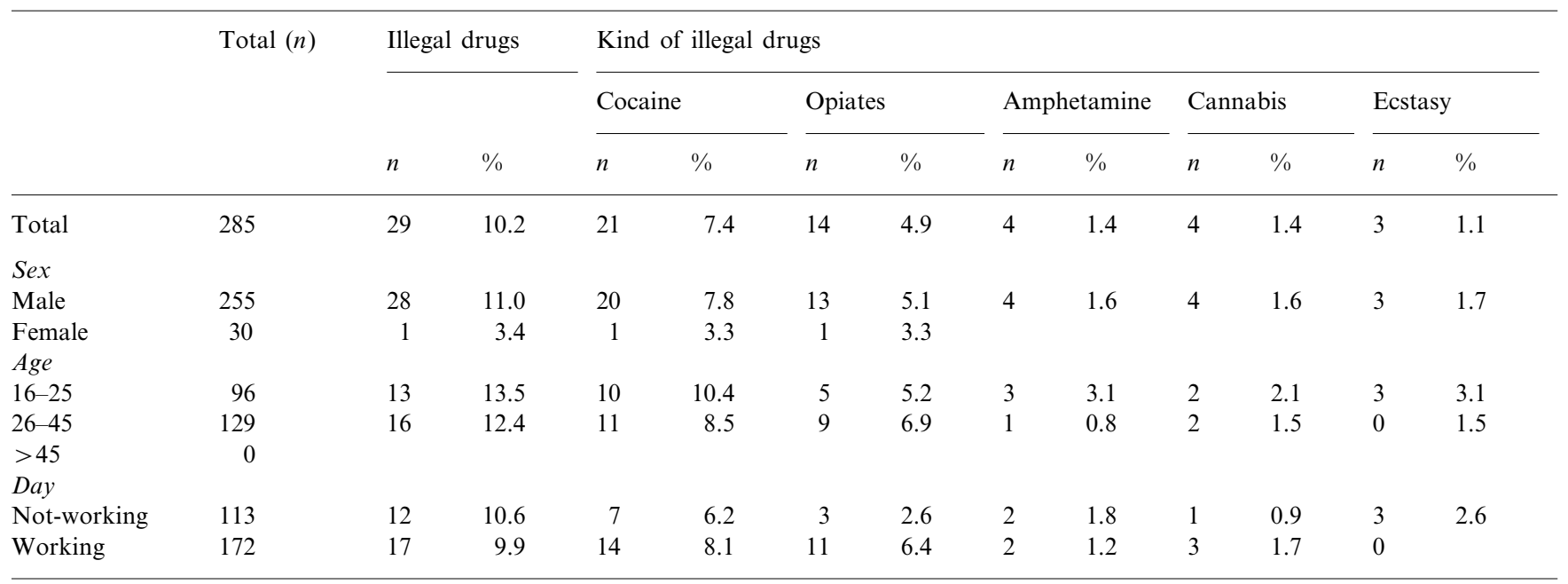


Presence of illegal drugs alone or with another substances in the blood of fatally injured drivers in Spain

\begin{tabular}{|c|c|c|c|c|c|c|c|c|c|c|}
\hline & \multicolumn{2}{|c|}{ Total } & \multicolumn{2}{|c|}{ Only with alcohol } & \multicolumn{2}{|c|}{$\begin{array}{l}\text { With alcohol and } \\
\text { other drugs }\end{array}$} & \multicolumn{2}{|c|}{ Only with other drugs } & \multicolumn{2}{|c|}{$\begin{array}{l}\text { Without other sub- } \\
\text { stances }\end{array}$} \\
\hline & $n$ & $\%$ & $n$ & $\%$ & $n$ & $\%$ & $n$ & $\%$ & $n$ & $\%$ \\
\hline Total & 46 & 100 & 9 & 19.6 & 13 & 28.3 & 20 & 43.5 & 4 & 8.7 \\
\hline Cocaine & 21 & 100 & 8 & 38.1 & 4 & 19 & 6 & 28.6 & 3 & 14.3 \\
\hline Cannabis & 4 & 100 & 0 & 0 & 2 & 50 & 2 & 50 & 0 & 0 \\
\hline Ecstasy & 3 & 100 & 0 & 0 & 3 & 100 & 0 & 0 & 0 & 0 \\
\hline
\end{tabular}

we have observed the frequent combined consumption of different substances including alcohol and medicines (benzodiazepines and analgesics-anti-inflammatory): In $91.3 \%$ of cases, these were detected together with another substance, with a mean in each sample of 2.2 . Also, in $68.9 \%$ of cases, some level-III (high-toxic) substance was detected.

It is obviously impossible to establish a causal relationship between the presence of illegal drugs in biological fluids and road traffic accidents, due in part to the number of confusing factors which play their part (speed, youth, combined use of other substances, etc.); however, what has indeed been revealed in some responsibility analysis studies is that those individuals who have taken illegal drugs and drive are at greater risk when it comes to being involved in a road accident (Drummer, 1994).

As for information available in Spain, there are few studies relating to drugs and accidents due to several reasons. First of all, samples (for legal purposes) are only taken to determine alcohol (or other drugs) in very few cases (generally in fewer than $3-5 \%$ of deaths). Secondly, because of what we colloquially refer to as the 'widow syndrome', that is, no attention is paid to whether the victim was under the influence of alcohol and/or other drugs, or not, it is preferred that the insurance companies pay the families of the victim and the incident be forgotten. Thus, a test for blood alcohol (and other drugs) is carried out only in cases in which the victim has shown clear signs of being under the effect of alcohol or other drugs. The fact that testing for alcohol in individuals killed or injured in road accidents has become a formal requirement in Spain (since December 1996), might constitute a means to put an end to this situation.

Recently (Alvarez et al., 1997) we presented a comparison of the data included in this paper (a determination of illegal drug concentration for non-legal purposes) with that obtained between 1992 and 1995 in the National Institute of Toxicology, concerning 979 people killed in road accidents (an analysis carried out for legal purposes). Data from both sources proved very similar: $10.2 \%$ of positive cases in our study, compared with $8.3 \%$ in the samples provided by the National Institute of Toxicology. In both cases cocaine and opiates represented the most commonly detected drugs.

Our data are consistent with those encountered in general at the population level: multiple drug taking is relatively common among those who abuse or are dependent and, the joint use of drugs with alcohol is harmful due to the fact that there is an increase in the negative effects that these substances have on driving ability. It is easy to state that the higher the drug concentration the more likely it is that the individual will be involved in a fatal road accident, as in the case of alcohol. However, as mentioned earlier (Consensus Development Panel, 1985; Road Safety Committee, 1996; Albery et al., 1998), we do not have enough information to support this.

As was indicated in the methodology, and with a view to establishing a ranking for the concentrations of different substances in biological fluids, three levels or degrees have been devised. Of the 29 samples in which some type of illegal drug was detected, in $68.9 \%$ of cases a level-III substance was found, corresponding to high, toxic or supratherapeutic levels. It should be pointed out in this regard that a close relationship exists between the amount of alcohol consumed, the plasma alcohol levels attained and the degree of impairment to psychomotor performance and road accident risk. It has not been possible to establish a relationship for other substances. At any rate, although the mere presence of the illegal drug (in medium or high-toxic concentration) does not allow us to establish this as a causal factor of the accident, the detection of high-toxic levels of drugs does suggest an increased likelihood of a road-traffic accident. In this regard, in some cases, drug concentration and risk of impairment have been shown; for instance, in Norway (Christophersen and Morland, 1997): in this country there is usually a clinical examination and blood sampling in drivers who were driving after having taken drugs, and the authors stated that there was a clear correlation between the degree of 
Quantifying illegal drug concentration in the blood of fatally injured drivers

\begin{tabular}{|c|c|c|c|c|c|c|c|c|}
\hline & \multicolumn{2}{|c|}{ Total illegal drugs } & \multicolumn{2}{|c|}{ Level-I } & \multicolumn{2}{|c|}{ Level-II } & \multicolumn{2}{|c|}{ Level-III } \\
\hline & $n$ & $\%$ & $n$ & $\%$ & $n$ & $\%$ & $n$ & $\%$ \\
\hline Cocaine & 21 & 100 & 13 & 61.9 & 5 & 23.8 & 3 & 14.3 \\
\hline Opiates & 14 & 100 & 8 & 57.1 & 1 & 7.1 & 5 & 45.7 \\
\hline Ecstasy & 3 & 100 & 3 & 100 & & & & \\
\hline Alcohol & 14 & 100 & 1 & 7.1 & 3 & 21.4 & 10 & 71.4 \\
\hline Medicines & 16 & 100 & 5 & 31.3 & 5 & 31.3 & 6 & 37.5 \\
\hline Total of samples with illegal drugs & 29 & 100 & 21 & 72.4 & 9 & 41.4 & 8 & 27.6 \\
\hline Total of samples with any substance & 29 & 100 & 3 & 10.3 & 6 & 20.7 & 20 & 68.9 \\
\hline
\end{tabular}

reasoning impairment observed in the clinical evaluation and the drug level detected in blood. Furthermore, the National Health Institute in Finland (Lillsunde, 1996) uses a three-level concentration range (low, therapeutic, high concentration) with a six-level estimation of impairing effects on driving ability (not probable, fairly improbable, cannot be excluded nor confirmed, cannot be excluded, quite probable, and probable). The study conducted by Drummer (1994), in which responsibility analysis and three-range drug concentrations were assessed, shows that those who had drug concentrations at medium or high level were more likely to be either responsible for or contributory to the accidents.

In our study we have only information about illegal drug concentration in the blood, and this does not allow us to establish the actual responsibility of the illegal drug in the accident. Nevertheless, when assessing the possible relationship between illicit drug concentrations and driving impairment/fatal accident, aspects like acute and chronic drug use, drug interactions and tolerance should be considered as well as drug concentration (Christophersen and Morland, 1997).

In addition, the detected medicines among those taking illegal drugs were benzodiazepines and analgesics. This is not surprising, since it is usual for habitual illegal drug takers to consume these kinds of medicaments. These findings coincide with those obtained in other reports (Gjerde et al., 1993; Harmeet et al., 1997).

Our study reveals the presence of illegal drugs among people killed in road accidents, as well as the high concentration in blood of illegal drugs and substances found in combination with them (alcohol and medicines). Cocaine was the most commonly detected drug.

\section{Acknowledgements}

Support for this study was provided by the Dirección General de Tráfico (National Traffic Agency), Ministerio del Interior (Home Office), Madrid, Spain.

\section{References}

Albery, I.P., Gossop, M., Strang, J., 1998. Illicit drugs and driving: a review of epidemiological, behavioural and psychological correlates. J. Subst. Misuse 3, 140-149.

Albery, I.P., Strang, J., 1995. Illicit drugs and driving. In: Taylor, J.F. (Ed.), Medical Aspects of Fitness to Drive. A guide for Medical Practitioners. The Medical Commission on Accident Prevention, London, pp. 142-156.

Christophersen, A.S., Morland, J., 1997. Drugged driving, a review based on the experience in Norway. Drug Alcohol Depend. 47, $125-135$

Alvarez, F.J., Sancho, M., Vega, J., Del Río, M.C., Rams, A., Queipo, D., 1997. Drugs other than alcohol (medicines and illicit drugs) in people involved in fatal road accidents in Spain. In: MercierGuyon, C. (Ed.), Proceedings of the 14th International Conference on Alcohol, Drugs and Traffic Safety T'97, vol. 2. CERMT, Annecy, pp. 677-682.

Budd, R.D., Muto, J.J., Wong, J.K., 1989. Drug of abuse found in fatally injured drivers in Los Angeles County. Drug Alcohol Depend. 23, 153-158.

Charlier, C., Plomteux, G., 1998. Alcohol, drugs, medication and highway safety in Belgium. Rev. Med. Liege 53, 25-28.

Consensus Development Panel, 1985. Drug concentration and driving impairment. J. Am. Med. Assoc. 257, 2618-2621.

De Gier, J., 1993. Driving Licenses and Known Use of Licit or Illict Drugs. Institute of Human Psychopharmacology, Maastrich.

De Gier, J., 1998. Road traffic and illicit drugs. Council of Europe, Strasbourg (P-PG/Circrout (98) 1).

Del Río, M.C., Alvarez, F.J., 1995a. Illegal drugs and driving. J. Traffic Med. 23, 1-5.

Del Río, M.C., Alvarez, F.J., 1995b. Illegal drug taking and driving: patterns of drug taking among Spanish drivers. Drug Alcohol Depend. 37, 83-86. 
Drummer, O.H., 1994. Drugs in drivers killed in Australian road traffic accidents: the use of responsibility analysis to investigate the contribution of drugs to fatal accidents. Monash University, Victoria.

Drummer, O.H., 1995. A review of the contribution of drugs in drivers to road accidents. In: The Effects of Drugs (Other than Alcohol) on Road Safety, First Report. Government Printer, Melbourne, pp. 1-28.

European Monitoring Centre for Drugs and Drug Addiction, 1999. Literature review on the relation between drug use, impaired driving and traffic accidents. European Monitoring Centre for Drugs and Drug Addiction, Lisbon (CT.97.EP.14).

Ferrara, S.D., Giorgetti, R., Zancaner, S., 1994. Psychoactive substances and driving: state of the art and methodology. Alcohol Drugs Driv. 10, 1-55.

Gjerde, H., Beylich, K.M., Morland, J., 1993. Incidence of alcohol and drugs in fatally injured car drivers. Accid. Anal. Prev. 25, 479-483.

Harmeet, S., Bjornstig, U., Eriksson, A., Ohman, U., Solarz, A., 1997. Drug and alcohol use among injured motor vehicle drivers in Sweden: prevalence, driver, crash, and injury characteristics. Alcohol Clin. Exp. Res. 21, 968-973.

Hansen, A.C., Kristensen, I.B., Dragsholt, C., Brangstrup Hansen, J.P., 1996. Alcohol and drugs (medical and illicit) in fatal road accidents in a city of 300000 inhabitants. For. Sci. Int. 79, 49-52.
Krüger, H.P., Bud, M.W., Huessy, F.B., Mettke, M., 1998. Illicit drugs in road traffic. Council of Europe, Strasbourg (P-PG/Circrout (98) 5).

Lillsunde, P., 1996. Drugs and driving. Analitical and epidemiological aspects. The National Public Health Institute, Helsinki.

Logan, B.K., Schwilke, E.W., 1996. Drug and alcohol use in fatally injured drivers in Washington State. J. Forensic Sci. 41, 505-510.

Merced, G.W., Jeffery, W.K., 1995. Alcohol, Drugs, and impairment in fatal traffic accidents in British Columbia. Accid. Anal. Prev., 27, 335-343.

Peat, M.A., Finkle, B.S., 1992. Toxicological assay of psychoactive substances in biological fluids. In: Ferrara, S.D., Giorgetti, R. (Eds.), Methodology in Man-Machine Interaction and Epidemiology on Drugs and Driving Safety. ARFI, Padova, pp. 95-109.

Rams, M.A., Ortega, A., Sancho, M., 1993. Influencia del alcohol y las drogas en los accidentes de tráfico. Rev. Esp. Drogodep. 178, $247-252$.

Road Safety Committee, 1996. Inquiry into the Effects of Drugs (Other than Alcohol) on Road Safety in Victoria, vol. 1, 2. Victorian Government Printer, Victoria.

Tedeschi, L, Frison, G, Castagna, F, Ferrara, S.D., 1992. Compresive EIA/GC screening and GS-MS confirmation of psychoactive substances and blood and urine. In: Ferrara, S.D., Giorgetti, R. (Eds.), Methodology in Man-Machine Interaction and Epidemiology on Drugs and Driving Safety. ARFI, Padova, pp. 147-185. 